\title{
STRUCTURE THEOREMS FOR GROUPS WITH DIHEDRAL 3-NORMALISERS
}

\author{
by N. K. DICKSON \\ (Received 14th March 1977)
}

\section{Introduction}

In this paper we prove five structure theorems for groups with dihedral 3normalisers. The interest in these theorems lies not so much in the results themselves as in what can be proved from them. The original versions of the results are contained in our doctoral thesis (1) where they are used to prove the following theorem, of which this paper, together with (2), (3) and other papers in preparation, will constitute a published proof:

Theorem. Let $G$ be a finite group with cyclic Sylow 3-subgroups. Let $d$ be an element of $G$ of order 3 and suppose that $N_{G}(\langle d\rangle)=\langle H, \tau\rangle$, where $(h \tau)^{2}=1$ for all $h \in H, H$ is abelian and 4 does not divide $|H|$. Suppose further that any simple group of order prime to 3 involved in $G$ is isomorphic to $S z(r)$ for some $r$. Then one of the following holds:

(a) 3 divides $|S(G)|$, in which case $G$ is soluble and $G=O_{2^{\prime}, 2}\left(O_{3^{\prime}}(G)\right) N_{G}(\langle d\rangle)$;

(b) $G / O_{2^{\prime}, 2}(G) \cong S L\left(2,2^{n}\right)$ for some integer $n \geqslant 2$;

(c) $G / O_{2}(G) \cong \operatorname{PSL}(2, q)$ where $q$ is a prime power, $q>5$ and $q \equiv \pm 5, \pm 7$ or \pm 11 $(\bmod 24)$

(d) $G / O_{2^{\prime}, 2}(G) \cong P S L(2, q)$ where $q$ is a prime power, $q>5, q \equiv \pm 5$ or $\pm 7(\bmod 24)$ and a Sylow 2-subgroup of $O_{2^{\prime}, 2}(G)$ has order 2;

(e) $G / O_{2^{\prime}, 2}(G) \cong P S L(2,7) \cong G L(3,2)$ and a Sylow 2-subgroup of $O_{2^{\prime}, 2}(G)$ is elementary abelian of order 8 ;

(f) $G / O_{2^{\prime}, 2}(G) \cong S_{5}$;

(g) $G$ has a subgroup $K$ of index 2 such that $G=K C_{G}(d)$ and $K / O_{2}(G) \cong$ $\operatorname{PSL}(2, q)$ where $q$ is a prime power, $q>5$ and $q \equiv \pm 5$ or $\pm 7(\bmod 24)$.

The five theorems proved in this paper are the following.

Theorem 1. Let $G$ be a finite soluble group with a normal subgroup $K$ of order prime to 3 such that $G / K \cong D_{6}$. Let $d$ be an element of $G$ of order 3 and suppose that $N_{G}(\langle d\rangle)=\langle H, \tau\rangle$, where $(h \tau)^{2}=1$ for all $h \in H, H$ is abelian and $H$ has a cyclic Sylow 2-subgroup. Then

$$
K=O_{2^{\prime}, 2}(K) C_{K}(d) \text {. }
$$

Theorem 2. Assume the same hypotheses as Theorem 1. Let $T$ be $a\langle d, \tau\rangle$ invariant Sylow 2-subgroup of $K$. Then $\left[T^{\prime},\langle d\rangle\right] \leqslant \mathrm{O}_{2}(K)$. 
Theorem 3. Let $G$ be a finite group with cyclic Sylow 3-subgroups. Let $d$ be an element of order 3 and suppose that $N_{G}(\langle d\rangle)=\langle H, \tau)$ where $(h \tau)^{2}=1$ for all $h \in H$ and $H$ is abelian. Suppose further that $G$ has a subgroup $V \cong V_{4}$ such that $\langle V, d\rangle \cong A_{4}$ and a soluble normal subgroup $K$ of order prime to 2 and 3 such that all involutions of $G / K$ are conjugate in $G / K$. Then $C_{K}(V)=1$.

(Note: this theorem is designed specifically to deal with a group $G$ with a normal subgroup $K$ such that $G / K \cong P S L(2, q), q$ odd.)

Theorem 4. Let $G$ be a finite group with a normal soluble subgroup $K$ of order prime to 2 and 3 such that $G / K \cong S L\left(2,2^{n}\right), n \geqslant 2$. Let d be an element of $G$ of order 3 and suppose that $N_{G}(\langle d\rangle)=\langle H, \tau\rangle$ where $(h \tau)^{2}=1$ for all $h \in H$ and $H$ is abelian. Then either

(a) $K=1$, or

(b) $n \leqslant 3$ and, if $T$ is a Sylow 2-subgroup of $G, C_{K}(T)=1$.

Theorem 5. Let $G$ be a finite group with a normal 2-subgroup $T$ such that $G / T \cong P S L\left(2, p^{n}\right), p>3, p^{n}>5$. Let $d$ be an element of $G$ of order 3 and suppose that $C_{T}(d)$ is cyclic. Then either

(a) $T \cong Z_{2^{m}}$ for some $m \geqslant 0$, or

(b) $T \cong Z_{2^{m}} \times Z_{2^{m}} \times Z_{2^{m}}$ for some $m \geqslant 1, G / T \cong P S L(2,7)$ and $G / T$ acts as $G L(3,2)$ on each elementary abelian section of $T$ of order 8.

Notation. Throughout this paper we use the notation of Gorenstein's book (4).

\section{Proof of Theorem 1}

Let $G$ be a minimal counterexample to Theorem 1 and let $K, H, d, \tau$ be as in the statement of the theorem.

Lemma 1.1. $O_{2}(K)=1$ and $\Phi\left(O_{2}(K)\right)=1$.

Proof. Immediate, since $G$ is a minimal counterexample.

Lemma 1.2. $K=O_{2,2^{2}}(K)$.

Proof. If $O_{2.2^{\prime}}(K)<K$ then $O_{2.2^{\prime}}(K)\langle d, \tau\rangle<G$ whence $O_{2 . z^{\prime}}(K)=O_{2}(K) C_{O_{2,2}(K)}(d)$. Let bars denote images under the natural map $G \rightarrow G / O_{2}(K)$. Then $d$ centralises $O_{2}(\bar{K})=\overline{O_{2,2^{\prime}}(K)}$. Thus $\left[O_{2^{\prime}, 2}(\bar{K}),(\bar{d})\right] \leqslant\left[O_{2,2}(\bar{K}), C_{\bar{G}}\left(O_{2^{\prime}}(\bar{K})\right] \leqslant O_{2^{\prime}, 2}(\bar{K}) \cap C_{\bar{G}}\left(O_{2^{\prime}}(\bar{K})\right)\right.$ $\leqslant O_{2^{\prime}}(\bar{K})$ by the Hall-Higman centraliser lemma. By (4, Theorem 6.2.2) $O_{2,2}(\bar{K}) \leqslant C_{G}(\bar{d})$, which is abelian. But $O_{2}(\bar{K})=1$. Therefore $O_{2^{\prime}, 2}(\bar{K})=O_{2}(\bar{K})$ which implies that $K=O_{2}(\bar{K})$, i.e. $K=O_{2,2}(K)$ contrary to assumption.

Lemma 1.3. $K=O_{2}(K) P$ for some prime $p$ and Sylow $p$-subgroup $P$ of $K$. 
Proof. For any odd prime $p$ dividing $|K|$ we may, by (4, Theorem 6.2.2), choose a Sylow $p$-subgroup $P$ of $K$ such that $O_{2}(K) P$ is $\langle d, \tau\rangle$-invariant. If $O_{2}(K) P<K$ then Theorem 1 applies to $O_{2}(K) P\langle d, \tau\rangle$, giving $P \leqslant C_{K}(d)$ whence $K=O_{2}(K) C_{K}(d)$, which is a contradiction.

Lemma 1.4. $P(d, \tau)$ acts irreducibly on $M=O_{2}(K)$ with $P$ acting faithfully.

Proof. $P$ certainly acts faithfully on $M$ by Lemma 1.1 and the Hall-Higman centraliser lemma. If $P\langle d, \tau\rangle$ does not act irreducibly on $M$ let $N$ be a composition factor of $M$, regarding $M$ as a $G F(2) P\langle d, \tau\rangle$-module. Then $N \neq M$. Let $G_{1}$ be the semidirect product $N . P\langle d, \tau\rangle$. By the minimality of $G, N . P=O_{2^{\prime}, 2}(N . P) C_{N . P}(d)$. Thus $[P,\langle d\rangle] \leqslant O_{z}(N . P) \leqslant C(N)$. This holds for all choices of $N$ so that $[P,\langle d\rangle] \leqslant C(M)$. But $P$ acts faithfully on $M$. Therefore $[P,\langle d\rangle]=1$, forcing $K=O_{2}(K) C_{K}(d)$, which is a contradiction.

Lemma 1.5. $C_{M}(d) \cong Z_{2}$.

Proof. $\quad C_{T}(d)$ is cyclic by hypothesis and $M$ is elementary. Therefore the lemma is true or $C_{M}(d)=1$. If the latter holds then (7, Corollary 3.2) gives us the usual contradiction that $K=O_{2}(K) C_{K}(d)$.

Lemma 1.6. $M$ is homogeneous as a $G F(2) P$-module.

Proof. We apply Clifford's Theorem (4, Theorem 3.4.1) to the action of $P\langle d, \tau\rangle$ on $M$ taking $P$ as the normal subgroup. Clearly $M$ has 6,3,2 or 1 homogeneous (or Wedderburn) components.

If there are six components we may write $M=V \oplus V d \oplus V d^{2} \oplus W \oplus W d \oplus W d^{2}$ where $W=V \tau$. Then, for any $v \in V, v+v d+v d^{2}$ is centralised by $d$ but not inverted by $\tau$, contrary to the structure of $N_{G}(\langle d\rangle)$.

If there are three components we may write $M=V \oplus V d \oplus V d^{2}$ where $V$ is a homogeneous component. Since $C_{M}(d) \cong Z_{2}$ it follows that $V \cong Z_{2}$. But then $P$ centralises $V$ whence $P$ centralises $M$, contrary to Lemma 1.4.

If there are two components then we may write $M=V \oplus V \tau$ where both $V$ and $V \tau$ are $\langle d\rangle$-invariant. Since $\tau$ has to invert $C_{M}(d), C_{V}(d)=C_{V \tau}(d)=1$. So $C_{M}(d)=1$, contrary to Lemma 1.5. Thus there is one component, which proves the lemma.

Lemma 1.7. $Z(P)$ acts trivially on $M$.

Proof. Let $N$ be an irreducible $P$-submodule of $M$. By Lemma $1.6 P$ acts faithfully and irreducibly on $M$. Thus $Z(P)$ is cyclic. But $Z(P)$ admits $\langle d, \tau\rangle$. So $Z(P)$ is centralised by $d$. But $C_{K}(d)$ is abelian and $C_{M}(d) \neq 1$. Therefore $C_{M}(Z(P)) \neq 1$. By the irreducibility of $M, Z(P)$ centralises $M$.

Since Lemmas 1.4 and 1.7 contradict each other we have proved Theorem 1. 


\section{Proof of Theorem 2}

Let $G$ be a minimal counterexample to Theorem 2 and let $K, H, T, d, \tau$ be as in the statement of the theorem. Note that $T$ exists for the following reasons. We can certainly choose a $d$-invariant Sylow 2-subgroup of $K$. Its normaliser will contain a Sylow 2-subgroup of $G$ and by a suitable conjugation in $N_{G}(\langle d\rangle)$ we obtain a $\langle d, \tau\rangle$-invariant Sylow 2-subgroup of $K$.

Lemma 2.1. $O_{2}(K)=1$.

Proof. Otherwise we could apply the theorem to $G / O_{2}(K)$ and deduce a contradiction.

Lemma 2.2. $K=O_{2^{\prime}, 2}(K)$.

Proof. By Theorem $1, K=O_{2^{\prime}, 2}(K) C_{K}(d)$. Since $C_{K}(d)$ is abelian $T \leqslant O_{2^{\prime}, 2}(K)$. If $O_{2^{\prime} \cdot 2}(K)<K$ then, by the minimality of $G,\left[T^{\prime},\langle d\rangle\right] \leqslant O_{2}\left(O_{2^{\prime}, 2}(K)\right) \leqslant O_{2}(K)$.

Lemma 2.3. $M=O_{2^{\prime}}(K)$ is an elementary abelian $p$-group for some prime $p(p \neq 2$ or 3) on which $T\langle d, \tau\rangle$ acts irreducibly and $T$ acts faithfully.

Proof. By Lemma 2.1 $T$ acts faithfully on $M$.

Suppose there exists a subgroup $L$ with $1 \neq L<O_{2}(K)$ and $L \triangleleft G$. By the minimality of $G,\left[T^{\prime},\langle d\rangle\right] \leqslant O_{2}(L T)$ and also $\left[T^{\prime},\langle d\rangle\right] L / L \leqslant O_{2}(K / L)$. Thus $\left[T^{\prime},\langle d\rangle\right]$ centralises $L$ and $O_{2^{\prime}}(K) / L$ whence $\left[T^{\prime},\langle d\rangle\right]$ centralises $O_{2^{\prime}}(K)$. This means that $\left[T^{\prime},\langle d\rangle\right]=1$, which is a contradiction. We conclude that no such subgroups $L$ exist, from which the lemma follows immediately.

Lemma 2.4. Either $M$ has 3 homogeneous components as $G F(p) T$-module or $C_{M}(d)=1$.

Proof. We apply Clifford's Theorem to the action of $T\langle d, \tau\rangle$ on $M$ with $T$ as the normal subgroup. Clearly $M$ has 6,3,2 or 1 homogeneous components.

If $M$ has six components we apply the argument of the six component case of Lemma 1.6 to obtain a contradiction.

If $M$ has two components the argument of the two component case of Lemma 1.6 shows that $C_{M}(d)=1$.

If $M$ has one component then $T$ acts faithfully and irreducibly on each irreducible $T$-submodule of $M$. Thus $Z(T)$ is cyclic, whence $Z(T) \leqslant C(d)$. Since $C_{M}(Z(T))$ is $T\langle d, \tau\rangle$-invariant and $T$ acts faithfully on $M, C_{M}(Z(T))=1$. But $C(d)$ is abelian and $Z(T) \leqslant C(d)$. We conclude that $C_{M}(d)=1$.

Hence either $M$ has three components or $C_{M}(d)=1$.

Lemma 2.5. $M$ has 3 homogeneous components as $G F(p) T$-module. Furthermore we may choose a component $V$ such that $V=V \tau$ and $M=V \oplus V d \oplus V d^{2}$. 
Proof. If $M$ does not have 3 components then by Lemma $2.4 C_{M}(d)=1$. Let $T_{1}$ be an abelian characteristic subgroup of $T$. Then, by (7, Corollary 3.2) applied to $M T_{1}\langle d\rangle$, $T_{1} \leqslant C(d)$ forcing $T_{1}$ to be cyclic.

We conclude that $T$ has no noncyclic abelian characteristic subgroups. By a theorem of $\mathrm{P}$. Hall (4, Theorem 5.4.9) $T^{\prime}$ is cyclic, whence $\left[T^{\prime},\langle d\rangle\right]=1$ which contradicts the hypothesis that $G$ is a counterexample. So $M$ has 3 components. The existence of $V$ follows immediately.

Lemma 2.6. $\quad C_{T}(d)=1$.

Proof. Since $v+v d+v d^{2} \in C_{M}(d)$ for any $v \in V$ and $C_{G}(d)$ is abelian $(v+v d+$ $\left.v d^{2}\right) x=v+v d+v d^{2}$ for all $x \in C_{T}(d)$. But $V, V d$ and $V d^{2}$ are all $T$-modules so that $v=v x, v d=v d x, v d^{2}=v d^{2} x$. Thus $x$ acts trivially on $M$, whence $x=1$. This proves that $C_{T}(d)=1$.

Lemma 2.7. $[\tau, T] \leqslant C_{T}(V)$.

Proof. For any $v \in V, \quad v+v d+v d^{2} \in C_{M}(d) \quad$ and $\quad$ so $\quad\left(v+v d+v d^{2}\right) \tau=$ $-v-v d-v d^{2}$. Since $V=V \tau$ it follows that $v \tau=-v$ so that $\tau$ is in the centre of the representation of $T\langle\tau\rangle$ on $V$. Thus $[\tau, T] \leqslant C_{T}(V)$.

Lemma 2.8. $\quad C_{T}(V) \cap\left(C_{T}(V)\right)^{d}=1$.

Proof. By Lemma $2.6 C_{T}(d)=1$. It follows from (4, Lemma 10.1.1 (ii)) that, if $t \in C_{T}(V) \cap\left(C_{T}(V)\right)^{d}, \quad t=\left(t^{d} t^{d^{2}}\right)^{-1} \in\left(C_{T}(V)\right)^{d^{2}} \quad$ so that $C_{T}(V) \cap\left(C_{T}(V)\right)^{d}=$ $C_{T}(V) \cap\left(C_{T}(V)\right)^{d} \cap\left(C_{T}(V)\right)^{d^{2}}$, which is trivial since it is the kernel of the action of $T$ on $M$.

Lemma 2.9. $T=\left\langle C_{T}(V),\left(C_{T}(V)\right)^{d}\right\rangle$.

Proof. Let $t \in T$ and let $u=\tau t^{-1} \tau$. By Lemma $2.7 \quad\left[\tau, u^{d^{-1}}\right]^{d}[\tau, u]^{-1} \in$ $\left\langle C_{T}(V),\left(C_{T}(V)\right)^{d}\right\rangle$. An easy calculation shows that this element is in fact $t^{d^{-1}} t^{-1}$. Because $C_{T}(d)=1(4$, Lemma 10.1.1) tells us that every element of $T$ can be written in the form $t^{d-1} t^{-1}$, whence the lemma.

Lemma 2.10. $\quad T^{\prime}=1$.

Proof. $\quad C_{T}(V) \triangleleft T$ and so, by Lemmas 2.8 and 2.9, $T=C_{T}(V) \times\left(C_{T}(V)\right)^{d}$ and hence also $T=\left(C_{T}(V)\right)^{d} \times\left(C_{T}(V)\right)^{d^{2}}=\left(C_{T}(V)\right)^{d^{2}} \times C_{T}(V)$. Thus $T=\left\langle\left(C_{T}(V)\right)^{d}\right.$, $\left.\left(C_{T}(V)\right)^{d^{2}}\right) \leqslant C\left(C_{T}(V)\right)$. Therefore $C_{T}(V)$ is abelian and hence so also is $\left(C_{T}(V)\right)^{d}$. Therefore $T$ is abelian.

We have now proved Theorem 2 because Lemma 2.10 contradicts the supposition that $G$ is a counterexample.

\section{Proof of Theorem 3}

Suppose Theorem 3 is false. Let $G$ be a minimal counterexample and let $d, \tau, H, K$ and $V$ be as in the statement of the theorem. Note first that since $K$ has odd order all 
involutions of $G$ are conjugate. Let $M$ be a non-trivial minimal normal subgroup of $G$ contained in $K, M$ is then an elementary abelian $p$-group for some prime $p \neq 2$ or 3 . Let $M^{*}=M \otimes_{G F(p)} \mathscr{K}$ where $\mathscr{K}$ is a field of characteristic $p$ containing a primitive 6-th root of unity.

$G$ acts on $M^{*}$ and $M^{*}$ is completely reducible as $\langle d, \tau\rangle$-module. Since $\tau$ inverts $C_{G}(d)$ it follows that $M^{*}$ has a basis such that $\langle d, \tau\rangle$ is represented by block diagonal matrices, each block being given by
(a) $d \rightarrow[1]$ $\tau \rightarrow[-1]$, or
(b) $d \rightarrow\left[\begin{array}{cc}\eta & 0 \\ 0 & \eta^{-1}\end{array}\right]$ $\tau \rightarrow\left[\begin{array}{ll}0 & 1 \\ 1 & 0\end{array}\right]$ where $\eta$ is a primitive cube root of unity.

If we let $a$ and $b$ be the dimensions of $M^{*}$ and $C_{M} \cdot(d)$ respectively, we see that $\operatorname{dim}\left(C_{M^{*}}(\tau)\right)=\frac{1}{2}(a-b)$.

Consider now the action of $\langle V, d\rangle$ on $M^{*}$. We may write $M^{*}=$ $C_{M^{*}}(V) \oplus M_{1} \oplus M_{2} \oplus \cdots \oplus M_{k}$ where $M_{1}, M_{2}, \ldots, M_{k}$ are irreducible $\langle V, d\rangle$-modules on which $V$ acts faithfully. Applying Clifford's Theorem to $M_{1}$ with $V$ as the normal subgroup we see that $M_{1}$ has 1 or 3 homogeneous components. If there is only one then $V$ acts faithfully on every irreducible $V$-submodule of $M_{1}$. Since $V$ is not cyclic this is impossible. Therefore $M_{1}$ has 3 components. The irreducibility of $M_{1}$ now implies that $M_{1}=N \oplus N d \oplus N d^{2}$ for some irreducible $V$-submodule $N$. $V / C_{V}(N)$ acts faithfully and irreducibly on $N$ and therefore $C_{V}(N) \cong Z_{2}$. Now $\left(C_{V}(N)\right)^{d}=C_{V}(N d)$ and $\left(C_{V}(N)\right)^{d^{2}}=C_{V}\left(N d^{2}\right)$. It follows that if $t \in V^{*}$ then $\operatorname{dim}\left(C_{M_{1}}(t)\right)=\frac{1}{3} \operatorname{dim} M_{1}$. Also, since $C_{M_{1}}(d)=\left\{n+n d+n d^{2}: n \in N\right\}, \operatorname{dim}\left(C_{M_{1}}(d)\right)=\frac{1}{3} \operatorname{dim} M_{1}$. Similarly for $M_{2}, \ldots, M_{k}$.

If we let $c=\operatorname{dim}\left(C_{M^{*}}(V)\right)$ then, since $t$ and $\tau$ are conjugate, $\operatorname{dim}\left(C_{M^{*}}(\tau)\right)=$ $\operatorname{dim}\left(C_{M^{*}}(t)\right)=c+\frac{1}{3}(a-c)$. Also $\operatorname{dim}\left(C_{M^{*}}(d)\right) \geqslant \frac{1}{3}(a-c)$. Comparing these with the earlier calculations we deduce that $c=0$. Therefore $C_{M^{*}}(V)=0$ and so $C_{M}(V)=1$.

$G$ is a minimal counterexample. We can therefore apply the theorem to $G / M$, obtaining $C_{K / M}(V M / M)=1$. We conclude that $C_{K}(V)=1$, which is a contradiction.

\section{Proof of Theorem 4}

We shall require the following lemma concerning $S L\left(2,2^{n}\right)$ for $n \geqslant 2$.

Lemma 4.1. Let $\langle a\rangle$ be a cyclic subgroup of $S L\left(2,2^{n}\right)$ of order $2^{n}-1$, and let $\langle b\rangle$ be a cyclic subgroup of $S L\left(2,2^{n}\right)$ of order $2^{n}+1$. Then $\left\{1, \tau, a^{k}, b^{l}: 1 \leqslant k \leqslant 2^{n-1}-1\right.$, $\left.1 \leqslant l \leqslant 2^{n-1}\right\}$ is a set of representatives for the conjugacy classes of $S L\left(2,2^{n}\right)$ and the character table of $\operatorname{SL}\left(2,2^{n}\right)$ is as follows:

\begin{tabular}{|c|c|c|c|c|}
\hline & 1 & $a^{k}$ & $\tau$ & $b^{\prime}$ \\
\hline$A$ & 1 & 1 & 1 & 1 \\
$B$ & $2^{n}$ & 1 & 0 & -1 \\
$C_{i}$ & $2^{n}-1$ & 0 & -1 & $-\left(\omega^{i l}+\omega^{-i l}\right)$ \\
$D_{i}$ & $2^{n}+1$ & $\theta^{j k}+\theta^{-j k}$ & 1 & 0 \\
\hline
\end{tabular}


where $\omega$ is a primitive $\left(2^{n}+1\right)$ th root of unity, $\theta$ is a primitive $\left(2^{n}-1\right)$ th root of unity, $1 \leqslant i \leqslant 2^{n-1}$, and $1 \leqslant j \leqslant 2^{n-1}-1$. Furthermore, the Brauer character of any irreducible $p$-modular representation of $S L\left(2,2^{n}\right)$, for $p \neq 2$ or 3 , is the restriction of an ordinary irreducible character to the $p$-regular elements.

Proof. The character table is well known; the calculations concerning the Brauer character are to be found in (8). [These are similar in nature to the calculations in (9, Proposition 3.1).]

Now let $G$ be a counterexample to Theorem 4 and let $K, T, d, \tau, n$ be as in the statement of the theorem.

Lemma 4.2. For some prime $p \neq 2$ or $3, G / K$ has an irreducible $p$-modular representation $M_{1}$ with the property that $m \tau=-m$ for all $m \in C_{M_{1}}(d)$.

Proof. Since $h^{\tau}=h^{-1}$ for all $h \in C_{K}(d)$, to obtain $M_{1}$ we take a suitable elementary abelian homomorphic image of $K$, tensor it with a large field of characteristic $p$ and then take an irreducible $G / K$-submodule.

Lemma 4.3. If $r=\operatorname{dim} M_{1}$ and $s=\operatorname{dim} C_{M_{1}}(d)$ and $\chi$ is the Brauer character of $M_{1}$ then

$$
\chi(1)=r, \quad \chi(d)=\frac{1}{2}(3 s-r), \quad \chi(\tau)=-s .
$$

Proof. $\quad M_{1}$ is completely reducible as $\langle d, \tau\rangle$-module and, using also Lemma $4.2, M_{1}$ therefore has a basis with respect to which $\langle d, \tau\rangle$ is represented by block diagonal matrices, each block being given by

(a) $d \rightarrow[1]$

(b) $d \rightarrow\left[\begin{array}{cc}\eta & 0 \\ 0 & \eta^{-1}\end{array}\right] \quad \tau \rightarrow\left[\begin{array}{ll}0 & 1 \\ 1 & 0\end{array}\right]$,

where $\eta$ is a primitive cube root of unity. Since $\eta+\eta^{-1}=-1, \chi(1)=r, \chi(d)=$ $s-\frac{1}{2}(r-s)=\frac{1}{2}(3 s-r)$ and $\chi(\tau)=-s$.

Lemma 4.4. $G / K \cong S L(2,8)$ or $S L(2,4)$.

Proof. An easy calculation using Lemma 4.1 shows that the Brauer character $\chi$ of Lemma 4.3 can only exist when $n=3$ and $\chi$ is the restriction of $C_{3}$ or when $n=2$ and $\chi$ is the restriction of $C_{i}$ for some $i$.

Lemma 4.5. $G / K \cong S L(2,8)$.

Proof. If not then by Lemma $4.4 G / K \cong S L(2,4)$. Since $S L(2,4)$ contains subgroups isomorphic to $A_{4}$ and $D_{6}$ and has all its involutions conjugate, $C_{K}(T)=1$ by Theorem 3, contrary to the supposition that $G$ is a counterexample.

Lemma 4.6. Let $M$ be an irreducible p-modular representation of $G / K$ for some prime $p \neq 2$ or 3. Suppose that $m \tau=-m$ for all $m \in C_{M}(d)$. Then $C_{M}(T)=1$. 
Proof. $G / K \cong S L(2,8)$. Note that the condition $m \tau=-m$ ensures that $M$ is non-trivial. By Lemma 4.4 we see that $M$ has as its Brauer character the restriction of $C_{3}$ to $p$-regular elements and therefore has dimension 7. Also $T \cong V_{8}$ and there is an element $a$ of order 7 in $N_{G / K}(T)$ acting regularly on $T^{*}$. Since $T$ acts faithfully on $M$, $M$ as a $T\langle a\rangle$-module has a composition factor $N$ on which $T$ acts faithfully. By (4, Theorem 3.4.3), $\operatorname{dim} N \geqslant 7$. Hence $M=N$, i.e. $M$ is irreducible as $T\langle a\rangle$-module. Thus $C_{M}(T)=1$.

Now let $G$ be a minimal counterexample to Theorem 4 .

Lemma 4.7. There is a prime $p$ for which $O_{p}(K) \neq 1$ and $O_{p}(K)=1$.

Proof. Since $K$ is soluble there is certainly a prime $p$ for which $O_{p}(K) \neq 1$. If $O_{p^{\prime}}(K) \neq 1$ then we may apply Theorem 4 to $G / O_{p}(K)$ and $G / O_{p^{\prime}}(K)$ to obtain $C_{K}(T) \leqslant O_{p}(K) \cap O_{p}(K)=1$, which is a contradiction.

Lemma 4.8. $K$ is a $p$-group.

Proof. If the lemma is false there exists a prime $q, q \neq p$, such that $q$ divides $\left|O_{p, p}(K)\right|$. Since $G / K \cong S L(2,8)$ we may let $b$ be an element of $G$ of order 9 such that $b^{3}=d$ and $\tau$ inverts $b$. Let $Q$ be a $\langle b, \tau\rangle$-invariant Sylow $q$-subgroup of $O_{p, p^{\prime}}(K)$. Then $Q$ acts faithfully on $O_{p}(K)$ and hence on $O_{p}(K) / \Phi\left(O_{p}(K)\right)$. Let $N$ be a subgroup of $O_{p}(K) / \Phi\left(O_{p}(K)\right)$ irreducible under the action of $Q\langle b, \tau\rangle$. Applying Clifford's Theorem to $N$ with $Q$ as the normal subgroup we find that there are $1,2,3,6,9$ or 18 homogeneous components.

If there are 2,6 or 18 components we can write $N=V \oplus V \tau$ where $V$ is $Q\langle b\rangle$-invariant. Since $\tau$ inverts $C_{N}(d)$ it follows that $C_{N}(d)=1$.

If there are 9 components we may choose a component $V$ such that $N=$ $V \oplus V b \oplus V b^{2} \oplus \cdots \oplus V b^{8}$. Clearly $C_{N}(b) \neq C_{N}(d)$. But $C_{K}(d)$ is abelian. So we should have $C_{N}(b)=C_{N}(d)$. Thus the 9 component case does not occur.

If there are 3 components we may choose a component $V$ such that $N=$ $V \oplus V b \oplus V b^{2}$ and $V d=V$. As remarked already $C_{N}(b)=C_{N}(d)$, whence $C_{N}(d)=1$.

If there is 1 component suppose that $Q / C_{Q}(N) \neq 1$. Then $Q / C_{Q}(N)$ acts faithfully and irreducibly on each irreducible $Q$-submodule of $N$. So $Z\left(Q / C_{Q}(N)\right)$ is cyclic. It admits $\langle b, \tau\rangle$ and is therefore centralised by $d$. If $C_{N}(d) \neq 1$ then since $C_{K}(d)$ is abelian $C_{N}\left(Z\left(Q / C_{Q}(N)\right)\right) \neq 1$. The irreducibility of $N$ now forces $Z\left(Q / C_{Q}(N)\right)$ to act trivially on $N$. This contradiction implies $C_{N}(d)=1$.

Thus in all cases $Q / C_{Q}(N)=1$ or $C_{N}(d)=1$. When $C_{N}(d)=1, Q / C_{Q}(N)$ is centralised by $d$ by (7, Corollary 3.2). So in all cases $Q / C_{Q}(N)$ is centralised by $d$. This holds for all choices of $N$, whence $Q \leqslant C(d)$. Thus $O_{p, p^{\prime}}(K) / O_{p}(K)$ is centralised by $d$ and therefore inverted by $\tau$. Since $C_{G / O_{p}(K)}\left(O_{p, p}(K) / O_{p}(K)\right) \triangleleft G / O_{p}(K)$ this contradicts $G / K \cong S L(2,8)$.

So $K$ is indeed a $p$-group.

Lemma 4.9. $C_{K}(T)=1$. 
Proof. Let $M$ be a non-trivial minimal normal subgroup of $G$ contained in $K$. Since $K$ is a $p$-group, $M \leqslant Z(K)$ and $G / K$ acts on $M$. The structure of $N_{G}(\langle d\rangle)$ ensures that $m \tau=-m$ for all $m \in C_{M}(d)$. By Lemma 4.6 $C_{M}(T)=1$.

Also, by applying Theorem 4 to $G / M, C_{K}(T) \leqslant M$. Whence $C_{K}(T)=1$.

Lemma 4.9 contradicts the definition of $G$; Theorem 4 is therefore proved.

\section{Proof of Theorem 5}

The following lemmas give properties of $\operatorname{PSL}(2, q)$, for $q=p^{n}, p$ prime, $p>3$, $p^{n}>5$, which we shall require. Let $\langle a\rangle$ and $\langle b\rangle$ be cyclic subgroups of $\operatorname{PSL}(2, q)$ of order $\frac{1}{2}(q-1)$ and $\frac{1}{2}(q+1)$ respectively, $d$ be an element of order $3, q_{1}$ and $q_{2}$ be representatives of the two conjugacy classes of $p$-elements of $\operatorname{PSL}(2, q)$, and $\omega$ and $\theta$ be primitive $\frac{1}{2}(q-1)$ and $\frac{1}{2}(q+1)$-th roots of unity respectively.

Lemma 5.1. If $q \equiv 1$ ( $\bmod 4)$, the ordinary character table of $\operatorname{PSL}(2, q)$ is as follows:

\begin{tabular}{|c|c|c|c|c|c|}
\hline & 1 & $q_{1}$ & $q_{2}$ & $a^{k}$ & $b^{\prime}$ \\
\hline$A$ & 1 & 1 & 1 & 1 & 1 \\
\hline$B$ & $\frac{1}{2}(q+1)$ & $\frac{1}{2}\left(1+V_{q}\right)$ & $\frac{1}{2}(1-\sqrt[V]{ } q)$ & $(-1)^{k}$ & 0 \\
\hline C & $\frac{1}{2}(q+1)$ & $\frac{1}{2}(1-\sqrt[V]{q})$ & $\frac{1}{2}(1+\sqrt[V]{q})$ & $(-1)^{k}$ & 0 \\
\hline$D$ & $q$ & 0 & 0 & 1 & -1 \\
\hline$E_{i}$ & $q-1$ & -1 & -1 & 0 & $-\left(\theta^{i l}+\theta^{-i l}\right)$ \\
\hline$F_{j}$ & $q+1$ & 1 & 1 & $\omega^{i k}+\omega^{-i k}$ & 0 \\
\hline
\end{tabular}

for $1 \leqslant i \leqslant \frac{1}{4}(q-1), 1 \leqslant j \leqslant \frac{1}{4}(q-5), 1 \leqslant k \leqslant \frac{1}{4}(q-1)$ and $1 \leqslant l \leqslant \frac{1}{4}(q-1)$. Furthermore, if $\chi$ is the Brauer character of an irreducible 2-modular representation of $\operatorname{PSL}(2, q)$, then $X$ is the restriction to the 2-regular elements of $A, B-A, C-A, E_{i}$ or $F_{j}$.

Proof. See Proposition 3.1 of (9).

In a similar manner we also obtain:

Lemma 5.2. If $q \equiv-1(\bmod 4)$, the ordinary character table of $\operatorname{PSL}(2, q)$ is as follows:

\begin{tabular}{|c|c|c|c|c|c|}
\hline & 1 & $q_{1}$ & $q_{2}$ & $a^{k}$ & $b^{j}$ \\
\hline$A$ & 1 & 1 & 1 & 1 & 1 \\
$B$ & $\frac{1}{2}(q-1)$ & $\frac{1}{2}(-1+V-q)$ & $\frac{1}{2}(-1-V-q)$ & 0 & $(-1)^{i+1}$ \\
$C$ & $\frac{1}{2}(q-1)$ & $\frac{1}{2}(-1-V-q)$ & $\frac{1}{2}(-1+V-q)$ & 0 & $(-1)^{i+1}$ \\
$D$ & $q$ & 0 & 0 & 1 & -1 \\
$E_{i}$ & $q-1$ & -1 & -1 & 0 & $-\left(\theta^{i l}+\theta^{-i l}\right)$ \\
$F_{j}$ & $q+1$ & 1 & 1 & $\omega^{j k}+\omega^{-j k}$ & 0 \\
\hline
\end{tabular}


for $1 \leqslant i \leqslant \frac{1}{4}(q-3), 1 \leqslant j \leqslant \frac{1}{4}(q-3), 1 \leqslant k \leqslant \frac{1}{4}(q-3)$ and $1 \leqslant l \leqslant \frac{1}{4}(q+1)$. Furthermore, if $\chi$ is the Brauer character of an irreducible 2-modular representation of $\operatorname{PSL}(2, q)$, then $\chi$ is the restriction to the 2-regular elements of $A, B, C, E_{i}$ or $F_{j}$.

Lemma 5.3. Let $M$ be a 2-modular representation of $\operatorname{PSL}(2, q)$ and let $\chi$ be its Brauer character. Then $\operatorname{dim}\left(C_{M}(d)\right)=\frac{1}{3}(\chi(1)+2 \chi(d))$.

Proof. Since $\operatorname{PSL}(2, q)$ has an involution inverting $d$ we may assume that $d$ is represented by the diagonal matrix $\operatorname{diag}\left\{1 \quad 1 \ldots 1 \quad \eta \eta^{-1} \eta \eta^{-1} \ldots \eta \eta^{-1}\right\}$ where $\eta$ is a primitive cube root of unity. Since $\eta+\eta^{-1}=-1, \quad \chi(d)=$ $\operatorname{dim}\left(C_{M}(d)\right)-\frac{1}{2}\left[\chi(1)-\operatorname{dim}\left(C_{M}(d)\right)\right]$, whence the lemma.

Lemma 5.4. Let $M$ be a 2-modular representation of $\operatorname{PSL}(2, q)$ in which $\operatorname{dim}\left(C_{M}(d)\right) \leqslant 1$. Then $\operatorname{dim}\left(C_{M}(d)\right)=1$ and either

(a) $M$ is the trivial representation, or

(b) $q=7$ and $\operatorname{dim} M=3$, or

(c) $q=11$ and $\operatorname{dim} M=5$.

Proof. Use Lemmas 5.1, 5.2 and 5.3, firstly to show that $\operatorname{dim}\left(C_{M}(d)\right)=0$ is impossible and then to list the cases with $\operatorname{dim}\left(C_{M}(d)\right)=1$. Cases (b) and (c) arise when the Brauer character of $M$ is the restriction of $B$ or $C$ to the 2-regular elements.

Lemma 5.5. Let $T$ be an elementary abelian 2-group on which $\operatorname{PSL}(2, q)$ acts irreducibly with $\left|C_{T}(d)\right| \leqslant 2$. Then $\left|C_{T}(d)\right|=2$ and either

(a) $T \cong Z_{2}$, or

(b) $T \cong Z_{2} \times Z_{2} \times Z_{2}, q=7$ and PSL $(2, q)$ acts as $G L(3,2)$ on $T$.

Proof. Since $\left|C_{T}(d)\right| \leqslant 2$ and $\operatorname{PSL}(2, q)$ has no 2-modular representations $M$ with $\operatorname{dim}\left(C_{M}(d)\right)=0$ by Lemma 5.4 , it follows that $\left|C_{T}(d)\right|=2$. By the same reasoning we can deduce that, if we tensor $T$ with a large field of characteristic $2, T$ remains irreducible. Thus, by Lemma $5.4,|T|=2$ or 8 (and $q=7$ ) or 32 (and $q=11$ ). The last of these cases is impossible because $\operatorname{PSL}(2,11)$ contains an element of order 11 and such an element cannot act faithfully on an elementary abelian group of order 32 . Finally we note that $\operatorname{PSL}(2,7) \cong G L(3,2)$.

We are now in a position to prove Theorem 5 . Let $G$ be a minimal counterexample and let us adopt the notation contained in the hypotheses of the theorem. We may choose a minimal non-trivial normal subgroup $T_{0}$ of $G$ contained in $T$.

Lemma 5.6. Either (a) $T_{0} \cong Z_{2}$ or (b) $T_{0} \cong Z_{2} \times Z_{2} \times Z_{2}, G / T \cong P S L(2,7)$ and $G / T$ acts as $G L(3,2)$ on $T_{0}$; either (c) $T / T_{0} \cong Z_{2^{m}}$ for some $m \geqslant 1$ or (d) $T / T_{0} \cong$ $Z_{2^{m}} \times Z_{2^{m}} \times Z_{2^{m}}$ for some $m \geqslant 1, G / T \cong P S L(2,7)$ and $G / T$ acts as $G L(3,2)$ on each elementary abelian section of $T / T_{0}$ of order 8 .

Proof. $T_{0} \leqslant \Omega_{1}(Z(T))$ so that $G / T$ acts irreducibly on $T_{0}$. The structure of $T_{0}$ now follows from Lemma 5.5. Since $G$ is a counterexample $T_{0} \neq T$ and the structure of $T / T_{0}$ is obtained by applying the theorem to $G / T_{0}$. 
Notation. Let $T_{1}$ be the inverse image in $T$ of $\Omega_{1}\left(T / T_{0}\right)$; when $G / T \cong P S L(2,7)$ let $x$ be an element of $G$ of order 7 .

Lemma 5.7. Parts (a) and (c) of Lemma 5.6 cannot occur together.

Proof. If (a) and (c) both held then $d$ centralises $T_{0}$ and $T / T_{0}$ and hence centralises $T$. Thus $T \cong Z_{2^{m+1}}$ which is a contradiction.

Lemma 5.8. Parts (a) and (d) of Lemma 5.6 cannot occur together.

Proof. Suppose (a) and (d) hold. Then $\left|C_{T_{1}}(d)\right|=4$ so that $C_{T_{1}}(d) \cong Z_{4}$. In particular $T_{1}$ contains an element of order 4 . But $x$ acts transitively on $\left(T_{1} / T_{0}\right)^{*}$. Therefore $T_{1}$ is a group with only one involution and hence is cyclic or quaternion, contrary to $T_{1} / T_{0} \cong Z_{2} \times Z_{2} \times Z_{2}$.

Lemma 5.9. Parts (b) and (c) of Lemma 5.6 cannot occur together.

Proof. Suppose (b) and (c) hold. As in the proof of Lemma $5.8 T_{1}$ contains an element of order 4. On the other hand, since $T_{0} \leqslant Z(T)$ it follows that $T_{1}$ is abelian, so that $\left\langle t^{2}: t \in T\right\rangle \cap T_{0} \cong Z_{2}$ which contradicts the minimality of $T_{0}$.

Lemma 5.10. Parts (b) and (d) of Lemma 5.6 hold.

Proof. Immediate from the three preceding lemmas.

Lemma 5.11. $T_{0}=\Omega_{1}(T)$.

Proof. Clearly $\Omega_{1}(T) \leqslant T_{1}$. If $T_{0} \neq \Omega_{1}(T)$ there is an involution $t$ in $T_{1} \backslash T_{0}$. So the coset $t T_{0}$ is a set of involutions. The transitivity of $x$ on $\left(T_{1} / T_{0}\right)^{*}$ now forces every element of $T_{1}^{*}$ to be an involution. Therefore $T_{1}$ is elementary abelian. But, as in the proof of Lemma 5.8, $T_{1}$ contains an element of order 4. We conclude that $T_{0}=\Omega_{1}(T)$.

Lemma 5.12. T is abelian or a Suzuki 2-group of order 64.

Proof. Use (6) since, by Lemma 5.11, $x$ permutes the involutions of $T$ transitively.

Lemma 5.13. T is a Suzuki 2-group of order 64.

Proof. Otherwise $T$ is abelian and by Lemmas 5.10 and $5.11 T$ satisfies the conclusions of Theorem 5 (b).

Lemma 5.14. T is not a Suzuki 2-group of order 64 .

Proof. If $T$ were a Suzuki 2-group of order 64 , then $T_{0}=\Phi(T)$ so that $\operatorname{PSL}(2,7)$ acts on $T / \Phi(T)$. However by (5) the only Suzuki 2-group of order 64 with 7 involutions, viz. $\Gamma_{9} 64 e$, has the property that its automorphism group induces on its 
Frattini factor a group of automorphisms of order 21 . This gives us the contradiction which completes the proof of the lemma and the theorem.

Acknowledgements. Parts of this paper are taken from the author's Oxford D.Phil. thesis. The author wishes to thank Dr W. B. Stewart, who supervised his work on that thesis, and also the Carnegie Trust for the Universities of Scotland, which provided financial support.

\section{REFERENCES}

(1) N. K. Dickson, D.Phil. thesis, University of Oxford (1975).

(2) N. K. Dickson, Conjugacy in groups with dihedral 3-normalisers, Glasgow Math. J. 18 (1977), 167-173.

(3) N. K. Dickson, 2-groups normalized by $S L\left(2,2^{n}\right)$, J. Algebra 47 (1977), 529-546.

(4) D. GoRenSteIN, Finite groups (Harper and Row, New York, 1968). 1964).

(5) M. HALl, JR., and J. SENIOR, The groups of order $2^{n}(n \leqslant 6)$ (Macmillan, New York,

(6) G. Higman, Suzuki 2-groups, Illinois J. Math. 7 (1963), 79-96.

(7) E. E. SHULt, On groups admitting fixed-point-free abelian operator groups, Illinois $J$. Math. 9 (1965), 701-720.

(8) W. B. Stewart, D. Phil. thesis, University of Oxford (1967).

(9) W. B. STEWART, Groups having strongly self-centralizing 3-centralizers, Proc. London Math. Soc. (3) 26 (1973), 653-680.

UNIVERSITY OF GLASGOW,

DEPARTMENT OF MATHEMATICS,

UNIVERSITY GARDENS,

GLASGOW,

SCOTLAND,

G12 8QW. 